\title{
Ultra-broadband optical signal processing using AIGaAs-OI devices
}

\author{
Galili, Michael; Da Ros, Francesco; Hu, Hao; Pu, Minhao; Yvind, Kresten; Oxenløwe, Leif Katsuo
}

Published in:

Optical Fiber Communication Conference 2017

Link to article, DOI:

10.1364/OFC.2017.Th1F.5

Publication date:

2017

Document Version

Peer reviewed version

Link back to DTU Orbit

Citation (APA):

Galili, M., Da Ros, F., Hu, H., Pu, M., Yvind, K., \& Oxenløwe, L. K. (2017). Ultra-broadband optical signal processing using AlGaAs-OI devices. In Optical Fiber Communication Conference 2017 (Vol. 2017). [Th1F.5] Optical Society of America (OSA). Optics Infobase Conference Papers https://doi.org/10.1364/OFC.2017.Th1F.5

\section{General rights}

Copyright and moral rights for the publications made accessible in the public portal are retained by the authors and/or other copyright owners and it is a condition of accessing publications that users recognise and abide by the legal requirements associated with these rights.

- Users may download and print one copy of any publication from the public portal for the purpose of private study or research.

- You may not further distribute the material or use it for any profit-making activity or commercial gain

- You may freely distribute the URL identifying the publication in the public portal 


\title{
Ultra-Broadband Optical Signal Processing using AlGaAs-OI Devices
}

\author{
Michael Galili, Francesco Da Ros, Hao Hu, Minhao Pu, Kresten Yvind, Leif K. Oxenløwe \\ DTU Fotonik, Technical University of Denmark, DK-2800 Kgs. Lyngby, Denmark \\ mgal@fotonik.dtu.dk
}

\begin{abstract}
Aluminum Gallium Arsenide on insulator (AlGaAs-OI) has recently been developed into a very attractive platform for optical signal processing. This paper reviews key results of broadband optical signal processing using this platform.

OCIS codes: (130.4310) Nonlinear; (190.4360) Nonlinear optics, devices; (060.4510) Optical communications
\end{abstract}

\section{Introduction}

Optical signal processing holds significant potential for bitrate-transparent processing for e.g. wavelength conversion, phase sensitive amplification and many other functionalities. Much effort has therefore been devoted to identifying attractive material platforms for nonlinear signal processing. Integrated solutions based on waveguides in nonlinear materials such as silicon [1], [2, 3] and silicon-germanium [4], [5] as well as silicon nitride [6] and high index doped glass [7] have all been investigated intensively for e.g. wavelength conversion. Unfortunately, the former two are affected by two photon absorption (TPA) at telecom wavelengths, while the latter two provide lower Kerr nonlinearity. In both cases the result is low efficiency of nonlinear interaction and signal generation. The use of second order nonlinearity in periodically poled lithium niobate [8] (PPLN) has yielded promising results. Integration of PPLN devices with other components is however very challenging as is very broadband operation of the nonlinear interaction.

In this paper we will discuss the AlGaAs-OI platform which, with proper dispersion and bandgap engineering, is not affected by the limitations mentioned above. We will outline the properties of the platform and key fabrication steps. We will then highlight three key demonstrations of nonlinear signal processing in AlGaAs. Firstly, we will summarize the first demonstration of optical wavelength conversion of 256 QAM data. Secondly we will outline the demonstration of high-performance integrated phase sensitive FWM. Finally, we will discuss high-quality spectral broadening enabling single-source $661 \mathrm{Tbit} / \mathrm{s}$ data transmission.

\section{The AlGaAs-OI Platform}

AlGaAs-OI is an attractive platform for nonlinear optical signal processing. Appropriate bandgap engineering can eliminate two-photon absorption (TPA) at telecom wavelengths (around $1550 \mathrm{~nm}$ ). This opportunity, combined with the possibility to fabricate high-quality integrated waveguide devices, positions AlGaAs-OI as one of the most promising platforms for integrating nonlinear optical signal processing.

Fig. 1 shows pictures of fabricated AlGaAs waveguides indicating typical dimensions and the structure of the devices. The AlGaAs-OI wafer is prepared by wafer growth, wafer bonding and substrate removal. The nanowaveguides are defined by electron-beam lithography and dry etching using a hydrogen silsesquioxane (HSQ) hard mask [9]. Fig. 1left shows a SEM image of the waveguide prior to $\mathrm{SiO}_{2}$ over-cladding deposition. The cross-section of the waveguide is $290 \times 630 \mathrm{~nm}^{2}$ and the propagation loss $1.5 \mathrm{~dB} / \mathrm{cm}$.
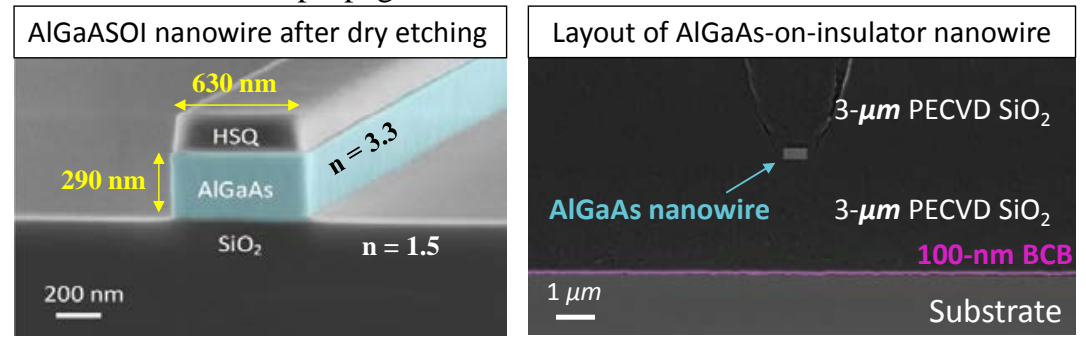

Fig. 1. - Left: SEM picture of a fabricated AlGaAsOI nano-waveguide (colored blue for clarity). Right: End-view of a fabricated waveguide after PECVD glass cladding. [9]

The AlGaAs core $(\mathrm{n} \approx 3.3)$ surrounded by insulator cladding $(\mathrm{n} \approx 1.5)$ creates a strong field confinement in the waveguide enhancing the already strong nonlinear Kerr effects in AlGaAs $\left(\mathrm{n}_{2} \approx 10^{-17} \mathrm{~W} / \mathrm{m}^{2}\right)$ [11]. Similar to other integrated platforms, the dispersion can be engineered through careful dimensioning of waveguide cross section. 
Inverse tapers [12] and lensed fibers are used in order to increase the coupling efficiency into the waveguide, reaching a coupling loss down to1.4 dB/facet.

\section{256-QAM wavelength conversion}

Fig. 2 shows a summary of the results of optical wavelength conversion of 10 Gbaud 256 QAM data signals using single pump FWM in a 9-mm long waveguide. This constitutes the first demonstration of 256 QAM optical wavelength conversion. It is seen from Fig. 2 (a) that wavelength conversion spanning close to $30 \mathrm{~nm}$ can be performed with less than $2 \mathrm{~dB}$ penalty in the OSNR required to reach a BER of $2 \times 10^{-2}$ after forward error correction (FEC) [13]. For a given pump power, and thus conversion efficiency, the idler OSNR scales linearly with the input signal power. Fig. 2 (b) and (c) shows the high achievable OSNR after wavelength conversion while Fig. 2 (d) shows a detailed characterization of the trade-off between increased OSNR and increased nonlinear distortion when increasing signal power launched into the waveguide. The increase in OSNR results in an improved pre-FEC BER only up to an input signal power of $9 \mathrm{dBm}$. Beyond this point self-phase modulation begins to dominate signal performance. A maximum achievable signal quality corresponding to an OSNR after conversion of $\sim 31 \mathrm{~dB}$ is found. For higher OSNR values the signal is degraded by nonlinear distortion reducing the signal quality. This is clearly recognized as the minimum of the red curve showing the pre-FEC BER in Fig. 2 (d). To achieve further improvement of the converted signal stronger pump power or higher accumulated nonlinearity in the waveguide is required.
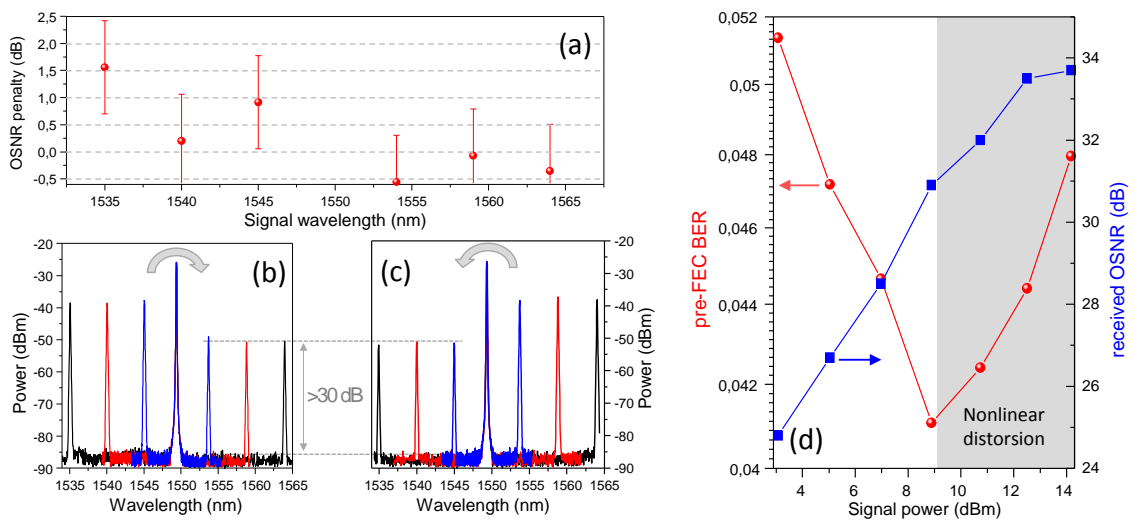

Fig. 2. - (a) OSNR penalty as a function of the signal wavelength for a post-FEC BER of $2 \times 10^{-2}$; (b) and (c) optical spectra at the waveguide output for signals on the short (b) and long (c) wavelength side of the pump. Idler OSNRs above $30 \mathrm{~dB}$ are shown for all the cases. (d) Tradeoff between increasing OSNR and nonlinear distortion. Figure is adapted from [13]

\section{Phase sensitive FWM}

Phase sensitive FWM is demonstrated in AlGaAs-OI achieving a high phase sensitive extinction ratio of $7.7 \mathrm{~dB}$ without the need for any enhancement through polarization filtering [14]. The phase-sensitive response can be observed by measuring the signal power at the output of the waveguide as the input signal phase is varied with respect to the pumps. Fig. 3 (a) shows the configuration of the optical waves for achieving phase sensitive FWM. The wavelength of the signal light is placed halfway between two stronger pumps and the interference with the idler light generated by FWM causes a net interaction of the waves which depends on the phase between signal and pumps.
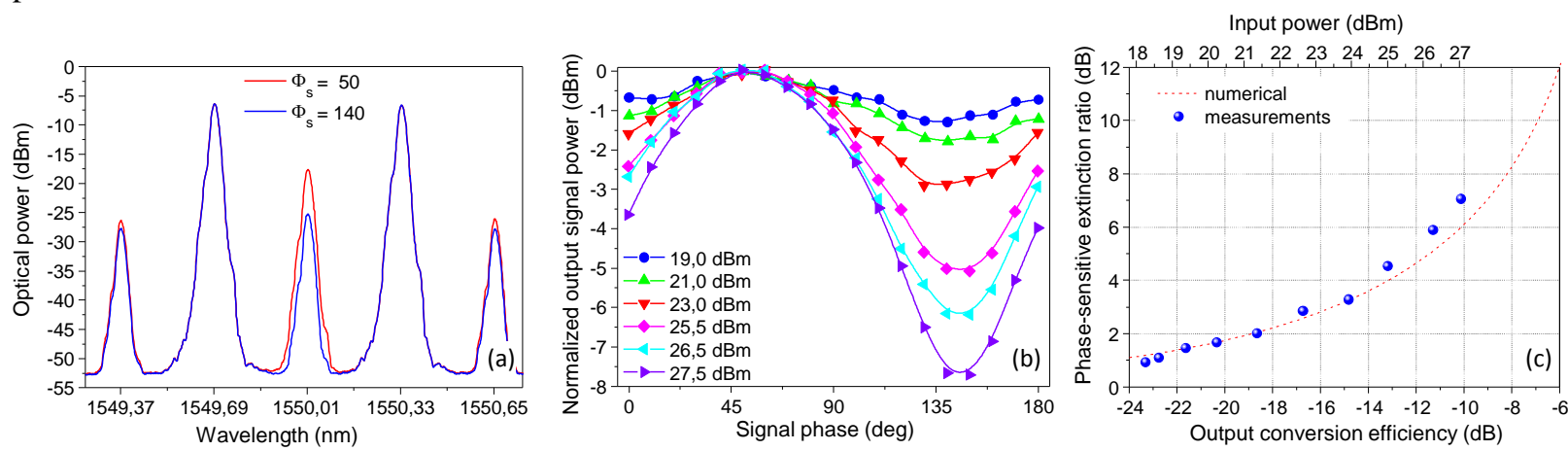

Fig. 3 - (a) Phase sensitive FWM for different signal phase $\Phi_{\text {s. }}$ (b) Phase sensitive extinction ratio measured at varying total launch powers. (c) Comparison between measured and calculated extinction ratio. Results are adapted from [10]. 
Fig. 3 (b) shows the normalized output signal power as a function of the signal phase for different input power levels into the AlGaAsOI waveguide. The sine-square shape of the curves shows the phase sensitive nature of the process. The phase sensitivity increases with power as the generated idler becomes stronger causing stronger interference with the original signal. This reaches a maximum of $7.7 \mathrm{~dB}$ for a total input power of $27.5 \mathrm{dBm}$. In Fig. 3 (c), the measured phase sensitive extinction ratio is compared to numerical calculations for changing output conversion efficiencies meaning different ratios between signal and idler waves. The numerical results are generated by solving the nonlinear Schrödinger equation using the split-step Fourier method. Consequently, they include contributions from higher-order FWM processes taking place in the waveguide. Good agreement is found between numerical and measured results, indicating that a further increase in conversion efficiency could enable even stronger phase sensitive interaction confirming the great potential of the AlGaAs-OI platform for this application.

\section{$661 \mathrm{Tbit} / \mathrm{s}$ signal source}

Finally, a narrow optical frequency comb seed is spectrally broadened in an AlGaAs-OI waveguide and used as a high-quality signal source for the generation of $661 \mathrm{Tbit} / \mathrm{s}$ data (80 ch. WDM, 16 QAM, 40 Gbaud, 30 cores) which is simultaneously transmitted over a single multicore fibre [15]. To accomplish this $10-\mathrm{GHz}$ pulses from a mode locked laser are amplified and launched into the AlGaAs waveguide with a peak power of 5.6 W (19.3 dBm average power). The strong nonlinearity of the waveguide broadens the spectrum of the light through self-phase modulation as seen in Fig. 4 (a) while maintaining a high OSNR of the individual lines. The OSNR is estimated to be $>40 \mathrm{~dB}$ at the center of the spectrum $(1552 \mathrm{~nm})$ and $\sim 30 \mathrm{~dB}$ in the worst part of the equalized spectrum shown in Fig. 4 (b) $(1563 \mathrm{~nm}$ ). This is sufficient to allow all parts of the spectrum to carry polarization multiplexed 16 QAM modulation at 40 Gbaud and be split 30 ways to fully load the 30-core fiber with identically modulated temporally de-correlated data channels.
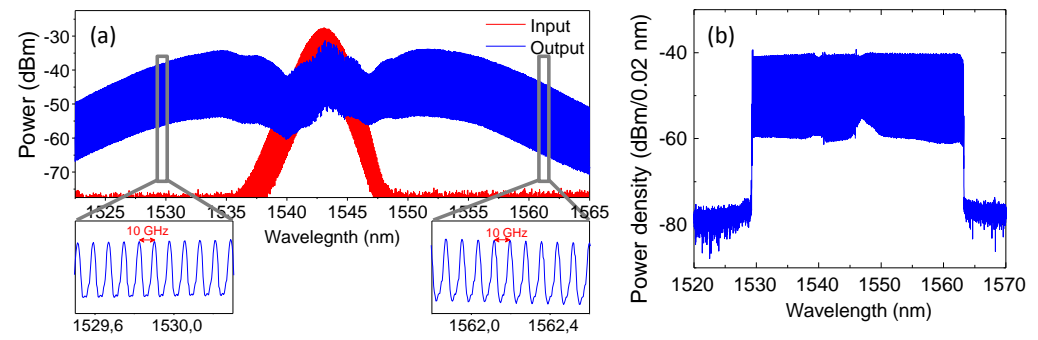

Fig. 4 - (a) Spectral broadening in AlGaAs waveguide due to SPM. (b) Spectrum after filtering and equalization. Figure is adapted from [15]

All the spectral and spatial channels simultaneously propagating in the fiber were characterized without making any changes to the transmitter. All channels could be detected error free and if the employed FEC is adapted to the channel performance a total net data rate of $661 \mathrm{Tbit} / \mathrm{s}$ was received. This is by far the highest amount of data transmitted using the light from a single integrated device and it shows the great potential of the AlGaAs-OI platform for use also in multi-frequency light sources for optical communication.

\section{Conclusions}

Through a number of record breaking achievements in optical signal processing the AlGaAs-OI platform has been confirmed as one of the most promising technologies for optical signal processing. As device fabrication research advances this platform is expected to significantly impact the role of nonlinear signal processing in communication systems and other high-performance applications.

\section{Acknowledgements}

This work was supported by the DNRF Research Centre of Excellence, SPOC (ref. DNRF123), and the Villum Foundation's Centre of Excellence, NATEC II.

\section{References}

[1] D. Vukovic et al., Opt. Express, Vol. 23, no. 3, p. 3640 (2015).

[2] F. Da Ros, et al., Opt. Express 22, 5029-5036 (2014).

[3] Y. Zhang, et al., Opt. Lett. 39, 363-366 (2014).

[4] M. A. Ettabib et al., Opt. Express, Vol. 21, no. 14, p. 16683 (2013).

[5] M. Ettabib, et al., in Proc. ECOC 2015, paper We3.6.5.

[6] C.J. Krückel et al., Opt. Lett., Vol. 40, no. 6, p. 875 (2015).

[7] F. Da Ros et al., Proc. OFC, Tu2K5, Anaheim (2016).

[8] T. Umeki, et al., Opt. Express 21, 12077-12084 (2013).
[9] L. Ottaviano et al. Opt. Lett. 41 pp.3396 (2016)

[10] F. Da Ros et al., IPC2016, WB1.1 (2016)

[11] M. Pu et al Optica 3 pp.823 (2016)

[12] M. Pu et al., Opt. Commun., 283, 3678-3682 (2010).

[13] F. Da Ros et al., in Proc. ECOC 2016, W.3.C.3 (2016)

[14] F. Parmigiani et al., JLT Vol. 33 No. 6, p. 1166 (2015)

[15] H. Hu et al., in Proc. CLEO 2016, paper JTh4C.1. 